\title{
ABUNDANCE JUMP IN THE INNER BULGES OF GALAXIES
}

\author{
O.K.SIL'CHENKO \\ Sternberg Astronomical Institute, University av. 13, 119899 Moscow.
}

\begin{abstract}
The metallicity difference of about an order between the kinematically decoupled nuclei and the surrounding bulges is found in 3 spiral galaxies possessing an axisymmetric potential in their centres.

Key words: Galaxies - Metallicity - Nuclei of galaxies
\end{abstract}

It is known that some galaxies, spirals (M 31, NGC 4594) and ellipticals (IC 1459, NGC 5813), have kinematically decoupled nuclei. From our study of gas kinematics in the inner parts of spiral galaxies we have found that almost $50 \%$ of our sample galaxies have decoupled nuclei, and half of these have fast rigid-body circular gas rotation [1]. The interesting question is if kinematically decoupled nuclei are distinguished by their stellar population properties from neighbouring bulges.

In 1989-90 we have investigated 12 bright nearby galaxies of various morphological types with the two-dimensional Multi-Pupil Fiber Spectrograph (MPFS) of the 6-m telescope of Special Astrophysical Observatory of RAS. In the spectral range 4800-5400 $\mathrm{A}$, with the spectral resolution 7-10 $\mathrm{A}$, the spectra from a rectangular area of 10" 11 " have been obtained for each galaxy (one spatial element is of $1.25 " \times 1.25 ")$. The direct image mode of MPFS has allowed us to construct isophotes for the central 30" of the galaxies under consideration, and the spectra have been used to derive line-strength profiles in the very centres of galaxies with rather high spatial resolution. The seeing quality during the observations was from 1 " to 2 ".

The analysis of the isophotes for NGC 615,7013 , and 7331 , previously known as possessing decoupled nuclei with circular gas rotation, has shown the coincidence of the major axis position angles for the very central isophotes and the outer disk ones. So these galaxies may have axisymmetric cores which are implied by their circular gas rotation. On the contrary, NGC 2655 and 7217 , suspecting to have strong non-circular gas motions in the centres, show significant twisting of the isophotes together with the increasing ellipticity in their nuclei, so they must have triaxial central components.

The analysis of the line-strength profiles has revealed a jump of the $M g I \lambda 5175$ absorption line equivalent width between the nuclei and the neighbouring bulges for 7 of 12 investigated galaxies, and among them there are distinct-nucleus galaxies NGC $615,7013,7331$. Using the broad-band colour $B-V$ of the bulges obtained earlier [4], I have compared distributions of the bulges and globular clusters (the data being from [2]) on the diagram $(M g, B-V)$ where effects of age and metallicity are separated [3]. The bulges have mostly the same position as the reddest globular clusters with $[\mathrm{Fe} / \mathrm{H}]-0.7--1.0$. So the jump of $M g I$-strength between the nuclei and the neighbouring bulges is a jump of metallicity reaching almost an order.

The behaviour of $M g I$-profiles in NGC 2655 and 7217 is rather complex, and the nuclei are not distinguished. It seems to be natural because a triaxial form of the central potential must result in strong radial gas flows which make impossible for a nucleus to possess an autonomous evolution. 


\section{Acknowledgements}

I am grateful to the Local Committee for the financial support giving me the possibility to attend the Symposium.

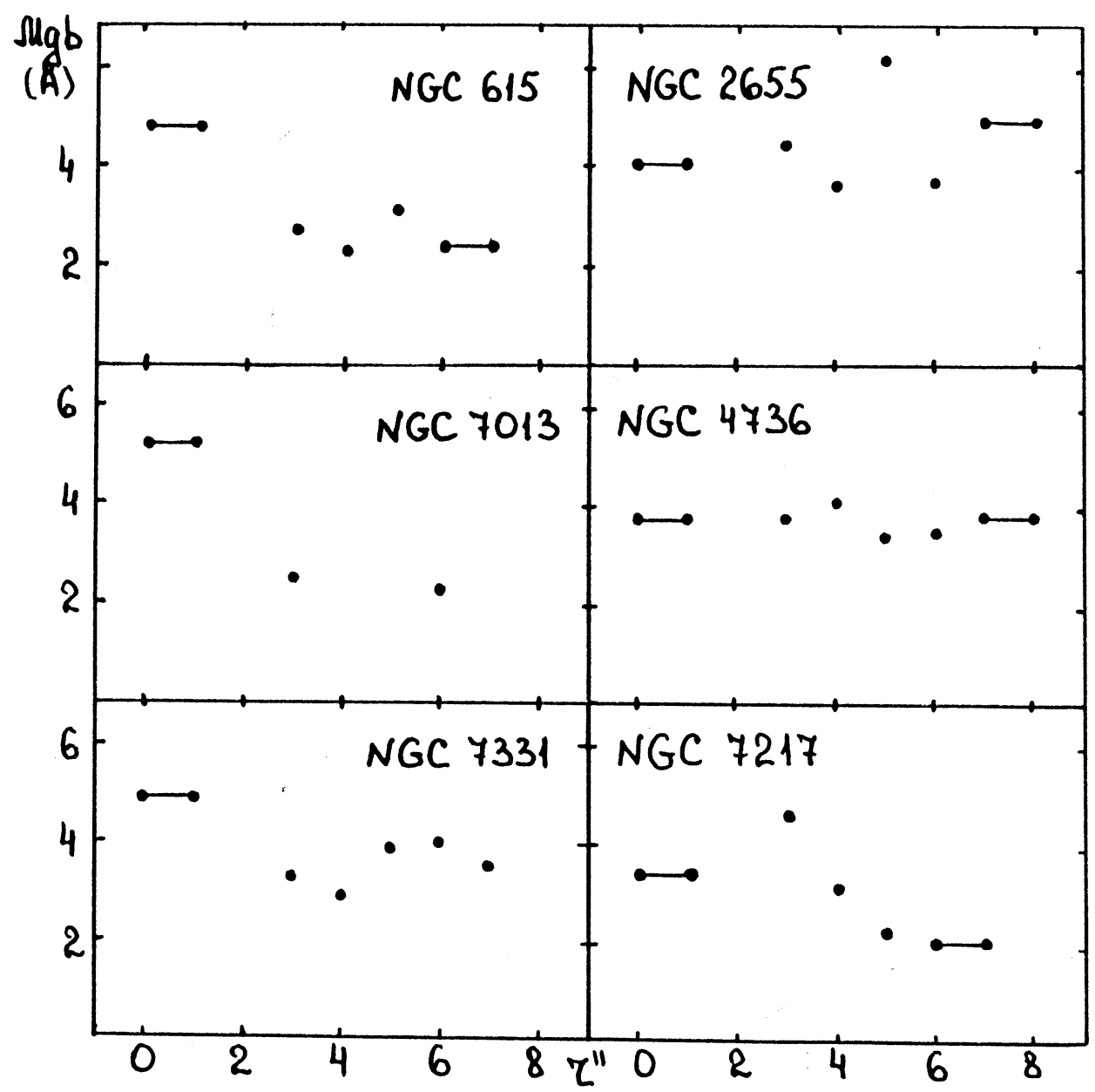

Fig. 1. The azimuthally-averaged magnesium line profiles for 6 spiral galaxies: NGC 615 , 7013 , and 7331 have an axisymmetric potential in the centres, NGC 2655, 4736, and 7217 have ovally distorted cores. The accuracy of equivalent widths is $0.3 \mathrm{~A}$.

\section{References}

[1] Afanasiev, V.L., Sil'chenko, O.K., Zasov, A.V., 1989. Astron. Astrophys., 213, L9.

[2] Burstein, D., et al. 1984. Astrophys. J., 287, 586.

[3] Sil'chenko, O.K., 1984. Pis'ma v Astron. Zh., 10, 19.

[4] Sil'chenko, O.K., 1989. Pis'ma v Astron. Zh., 15, 493. 